\title{
La investigación de la actividad pesquera y marítima: un análisis a través de las revistas españolas de Geografía (1950-2015)
}

An investigation into fishing and maritime literature: an analysis of Spanish geographic journals (1950-2015)

\author{
María Ángeles Piñeiro Antelo \\ manxeles.pineiro@usc.es \\ Rubén Camilo Lois González \\ rubencamilo.lois@usc.es \\ Departamento de Geografía \\ Universidad de Santiago de Compostela (España)
}

\section{Resumen}

En este trabajo se realiza un estudio bibliométrico de los artículos publicados en diez revistas geográficas españolas, en tanto son consideradas el principal medio de difusión de la investigación realizada por geógrafos en España, con el objetivo de evaluar las aportaciones relacionadas con el estudio de las actividades pesqueras y marítimas, con el marco espacial, jurídico y normativo en que se desarrollan, y relativos a la gobernanza del mar y de los espacios litorales. Se destaca que la temática abordada aparece concentrada en unas pocas revistas, en unas determinadas líneas de investigación, y en un reducido número de autores. Esta situación es general en todas las regiones españolas, incluso en aquellas en donde estas actividades tienen un gran peso socioeconómico. Se avanzan una serie de conclusiones relativas a los motivos causantes de esta situación.

Palabras clave: investigación; litoral; comunidades costeras; revistas científicas españolas. 


\begin{abstract}
This article presents a bibliometric study of the production published in ten Spanish Geographic journals, that are considered the main means of diffusion of the investigation carried out by geographers in Spain. In particular, we consider geographic research related to the study of coastal communities, the organization of their societies, and the characterization of the activities related to the sea and the coastal spaces. We emphasize that this theme is concentrated in a few journals, in certain lines of research, and in a small number of authors. This situation is common in all the Spanish Regions, even in those where these activities have a great socio-economic weight. We advance a series of conclusions regarding the causes of this situation and propose some work topics.
\end{abstract}

Key words: research; littoral; fishing; coastal communities; Spanish scientific journals.

\title{
1 Introducción
}

El objetivo de este trabajo es evaluar la importancia del mar, la pesca, y sus aspectos relacionados como tema de estudio en la Geografía española. Se analiza la literatura científica publicada en revistas geográficas para conocer cómo esta disciplina ha abordado estas temáticas desde mediados del siglo XX hasta el momento actual. Para realizar el estudio se ha hecho una selección entre las más de cincuenta revistas geográficas que, con carácter periódico, se publican en España. Nos hemos centrado en diez de ellas, que han sido elegidas en función de su antigüedad, importancia científica y predicamento entre los geógrafos españoles. También se ha considerado como elemento de selección el área geográfica o universidad en la que se publican, en el intento de incluir revistas de los ámbitos territoriales en los que la pesca constituye tradicionalmente una actividad económica relevante.

Cuando hablamos de la pesca estamos a considerar un conjunto de actividades que en sentido amplio conforman el sector. En nuestro análisis incluimos la pesca extractiva y el marisqueo, pero también la acuicultura, el procesado y conservación de productos de la mar, y su comercialización final. De manera paralela, también son objeto de estudio en este trabajo las publicaciones relacionadas con las políticas de ordenación y protección de recursos pesqueros, y de planificación, gestión, y gobernanza del espacio marítimo por un lado, y con los puertos y las actividades portuarias, indirectamente afectados por la pesca, por otro.

La importancia de esta temática marítimo-pesquera en relación a otras líneas de investigación, y sus enfoques dominantes, ha sido una cuestión que ha preocupado a un número significativo de geógrafos en los últimos 50 años, hasta el punto de convertirse en un tema de trabajo en sí mismo (Suárez de Vivero, 1979; Pérez, 1996; Durán et al., 1997; Lois, 1999; Santos, 2001b). La debilidad de los estudios geográficos relacionados con el mar, las motivaciones por las cuales el 
mar ha tenido un tratamiento poco cohesionado en la investigación geográfica, y las reacciones de la Geografía ante el fuerte desarrollo de las Ciencias del Mar son temas directamente relacionados con el cuestionamiento de la pertenencia del espacio marítimo al ámbito geográfico. El desarrollo de las teorías que identificaban el espacio geográfico con espacio habitado contribuyó de manera clara a la pobre aportación de artículos, ponencias y comunicaciones de temática marítima a las principales publicaciones y eventos de carácter geográfico, tanto en España como en el resto de Europa, durante el siglo XX (Suárez de Vivero, 1979).

Es a partir de la década de 1970 en que la bibliografía geográfica española iría incorporando aspectos oceánicos de gran impacto en ese momento, relativos al estado de los recursos, o a la extensión de los límites jurisdiccionales marítimos. Esta incorporación progresiva, condujo a alcanzar un cierto desarrollo, en los últimos años del siglo XX y en los primeros del XXI, de los trabajos geográficos centrados en estudios regionales sobre puertos, y en el impacto de la entrada de España en la CEE en el sector pesquero. En este período también se celebraron un número significativo de congresos y jornadas relativos a las comunidades pesqueras, y prosperaron los intercambios científicos entre geógrafos españoles, noruegos y canadienses trabajando en temas pesqueros. El auge de los estudios relacionados con la actividad pesquera tuvo su mayor exponente entre los años 2005 y 2007 en el Departamento de Geografía de la Universidad de Santiago, desde donde se organizaron una serie de reuniones científicas que actuaron como catalizadores de las contribuciones geográficas sobre esta temática. Destacamos las tres ediciones del congreso internacional "Globalización, Arraigo Geográfico y Comunidades Costeras en tiempos de cambio", coorganizadas con la Universidad de Bergen, o el congreso "Canadá y la Unión Europea, unidos por el mar. La gestión de los océanos en el siglo XXI". A estos eventos se sumaron cursos de verano, jornadas y seminarios con el mar y las actividades pesqueras como marco de trabajo. Aun así, y después de esta eclosión, en la primera década del presente siglo, de reuniones, cursos, congresos y exposiciones, en los últimos años se ha regresado a una cierta atonía en esta línea de investigación, que tan dinámica se había mostrado unos años antes.

\section{Metodología}

Para abordar el impacto de la pesca y las actividades marítimas como tema de estudio geográfico se hecho una selección entre aquellas revistas geográficas españolas de más larga trayectoria, y con mayor consideración entre los geógrafos españoles. La principal vía de difusión de la investigación geográfica publicada en España la constituyen las revistas científicas, asociadas a departamentos universitarios de geografía. Se ha intentado realizar un estudio prolongado en el tiempo, necesario para detectar cambios de tendencias, de temáticas de estudio y de enfoques dominantes, que se inicia el año de aparición de cada revista y abarca hasta el fin del año 2015. 
Ante la dificultad de analizar todas las revistas españolas, nos hemos centrado en diez, algunas de ellas de clara vocación internacional, incluidas en índices de citación nacionales e internacionales, y que se encuentran entre las mejor puntuadas por la Comisión Nacional Evaluadora de la Actividad Investigadora (CNEAI), y en otras con una orientación más regionalista. Para completar esta selección también se ha tenido en cuenta el índice IN-REC elaborado hasta 2010 por el Grupo de Investigación sobre Evaluación de la ciencia y de la comunicación científica de la Universidad de Granada. Hemos seleccionado, entre las revistas españolas de Ciencias Sociales-Geografía, cuatro que se encuentran en el primer cuartil, ${ }^{1}$ tres del segundo cuartil ${ }^{2}$ y dos del tercer cuartil. ${ }^{3}$ Finalmente, hemos incluido en el análisis a la revista Semata, de la Facultad de Geografía e Historia de la Universidad de Santiago de Compostela, por la importancia que tiene en la difusión de la producción científica del Departamento de Geografía de dicha universidad, uno de los más atentos en España al estudio de la las actividades pesqueras a diferentes escalas, por el gran peso que el sector de la pesca tiene en Galicia desde el punto de vista socioeconómico.

La mayor parte de los números de las revistas que forman parte de esta selección están disponibles en formato electrónico, lo que hace más ágil y rápido el acceso a ellos. La consulta en biblioteca ha completado el proceso en aquellos números, esencialmente los más antiguos, de los que no existe copia digital. Debemos puntualizar que, ante la imposibilidad de leer todos los artículos que estamos considerando, fundamentalmente por motivos de tiempo, ya que se trata de más de 7000 aportaciones, hemos realizado una aproximación al contenido de los mismos a partir del análisis de su título en todos los casos. De manera complementaria, hemos analizado el resumen y las palabras clave en aquellos casos en los que estaban disponibles.

1. Revista Estudios Geográficos, fundada en 1940 por el Instituto Juan Sebastián Elcano del CSIC, publica artículos dedicados a la investigación científica en geografía, pero también noticias, comentarios y reseñas bibliográficas. Ha sido trimestral hasta 2004, y a partir de entonces se publican dos números al año. Está dirigida por Fernando Arroyo llera, de la Universidad Autónoma de Madrid

2. El Boletín de la Asociación de Geógrafos Españoles, trimestral desde 2018, es editado por la Asociación de Geógrafos Españoles (AGE), y publicó su número uno en el año 1984. Las contribuciones se distribuyen en varios apartados, categorizados en artículos, notas de síntesis, notas de libros y tesis doctorales. En la actualidad la editora jefa es Pilar Paneque Salgado, de la Universidad Pablo de Olavide.

1 Scripta Nova, Boletín de la Asociación de Geógrafos Españoles, Cuadernos de Geografía de la Universidad de Valencia y Estudios Geográficos.

2 Documents d'Anàlisi Geogràfica, Anales de Geografía de la Universidad Complutense y Ería.

3 Investigaciones Geográficas y Cuardernos Geográficos de la Universidad de Granada. 
3. La Revista Ería, editada por el Departamento de Geografía de la Universidad de Oviedo, apareció en 1980, y se publica en la actualidad con carácter cuatrimestral. Su director es Felipe Fernández García, miembro de este departamento.

4. La Revista Anales de Geografía de la Universidad Complutense, fue anual desde 1981 hasta 2006, y semestral desde 2007. Recoge artículos relacionados con el análisis e interpretación del espacio geográfico español, con especial atención al entorno regional de Madrid. Está editada por los Departamentos de Geografía Humana, y de Análisis Geográfico Regional y Geografía Física de la Universidad Complutense de Madrid. Desde el año 2007 aparece una sección en la revista denominada "La Geografía en el mundo", focalizada en América Latina. Su director es José Antonio Sotelo Navalpotro, catedrático de Análisis Geográfico Regional en la Universidad Complutense de Madrid.

5. La Revista Investigaciones Geográficas, del Instituto Universitario de Geografía de la Universidad de Alicante, empezó a publicarse en 1983. En la actualidad está dirigida por Antonio Manuel Rico Amoros, del Departamento de Análisis Geográfico Regional y Geografía Física. Hasta 1994 su periodicidad fue anual, y a partir de ese momento, y hasta 2001, semestral. Entre 2002 y 2011 aparecieron tres números al año, que se reducirían a dos entre 2012 y 2015.

6. Revista Documents d'Anàlisi Geogràfica, de las Universidades de Girona, y Autónoma de Barcelona, que se publica desde 1982, con carácter semestral. Da cabida a trabajos de diversas temáticas, desde la planificación territorial, hasta la epistemología de la Geografía. Predominan los estudios sobre Cataluña pero también aparecen publicados trabajos sobre otros territorios. Se estructura en dos secciones: artículos, y notas y documentación. Esta revista supone la fusión y continuación de otras tres: Documents d'Anàlisi Urbana, Documents d'Anàlisi Territorial y Documents d'Anàlisi Metodològica. Sus editores son Juan Antonio Mónedes Cabrerizo, Isabel Salamaña Serra y Miguel Solana Solana.

7. La revista Scripta Nova es una publicación electrónica de Geografía y Ciencias Sociales de la Universidad de Barcelona, que inicia su andadura en 1997, y que se difunde exclusivamente en formato digital. Su director es Horacio Capel, catedrático de Geografía Humana. Más de un tercio de su producción son artículos centrados en el ámbito iberoamericano, y es una de las revistas españolas de mayor difusión en países de habla española y portuguesa.

8. Cuadernos de Geografía, de la Universidad de Valencia, publicada desde el año 1964. Su principal objetivo es la difusión de artículos de investigación referida al territorio valenciano o a un ámbito espacial superior. También incluye aportaciones teóricas y metodológicas sobre la especialidad geográfica. Su periodicidad es semestral y su director es el catedrático Juan Mateu Bellés. 
9. Cuadernos Geográficos, de la Universidad de Granada, es una revista científica fundada en 1971, que publica trabajos de interés geográfico centrados especialmente en Andalucía y España, aunque también en Latinoamérica y en los países mediterráneos. Tiene una periodicidad anual y sus directores son Carmen Egea Jiménez y Francisco Antonio Navarro Valverde, del Departamento de Geografía Humana.

10. Semata: ciencias sociales y humanidades. Es la revista anual de la Facultad de Geografía e Historia de la Universidad de Santiago de Compostela, y lleva publicados veintisiete números entre 1988 y 2015. Su estructura tiene un carácter monográfico y rotatorio entre las áreas de Geografía, Historia e Historia del Arte. Su directora es Ana Mª Suárez Piñeiro, historiadora de la Universidad de Santiago de Compostela.

Al estudio de esta decena de revistas se añadió el de la Revista Española de Estudios Agrosociales y Pesqueros, publicada por el Ministerio de Agricultura y Pesca, Alimentación y Medio Ambiente desde 1998, y que es continuadora de la Revista de Estudios Agrosociales, publicada entre 1952 y 1994, y Agricultura y Sociedad, publicada entre 1976 y 1998. Esta revista fue seleccionada por su clara orientación temática, especializada en el medio rural con referencia especial a los sectores agrario, pesquero y forestal, abordados desde las Ciencias Sociales. Resulta relevante el hecho de que nazca por refundación de otras revistas españolas, y pase a incluir en su título la referencia a los estudios pesqueros.

Finalmente, para completar este proceso de análisis, se consideraron una serie de monográficos dedicados al estudio de la pesca por parte de revistas científicas españolas, aunque ninguna de ellas posea un carácter exclusivamente geográfico. Nos referimos a la revista Información Comercial Española, publicada por el Ministerio de Economía, que dedicó el n 546 de 1979 a la política pesquera. Este número se organizó en cuatro apartados: estructura del sector pesquero, política internacional pesquera, política nacional pesquera, y bibliografía. En segundo lugar, citamos la revista Situación, publicada por el Servicio de Estudios del Banco de Bilbao. El número 4 de 1984 se centra en el estudio de "La pesca española ante el reto de futuro". Finalmente la revista El Campo, también publicada por el Banco de Bilbao, y ya desaparecida desde 1996, dedicó su n 99 de 1985 al "Pasado, presente y futuro de la pesca".

Después de analizar el título, y para los casos disponibles, el resumen, y las palabras clave de las contribuciones presentadas a las revistas españolas citadas, pasamos a exponer de manera detallada los resultados obtenidos.

\section{Resultados}

El análisis de las diez publicaciones citadas, que en conjunto suman un total de 7210 aportaciones, deja unos resultados muy pobres en relación al estudio geográfico de las actividades marítimo 
pesqueras, ya que solo 70 de ellas se ajustan a la temática considerada. Una producción muy exigua teniendo en cuenta el amplio período estudiado, y la importancia de la economía pesquera a nivel estatal. Si exceptuamos la revista gallega Semata, con un número monográfico dedicado al mar, y la revista Estudios Geográficos, del CSIC, en ninguna de las revistas analizadas la temática marítima alcanza el $1 \%$ de su producción. En relación a temas y autores, hay una clara especialización en dos grandes temáticas: las actividades pesqueras y las actividades portuarias. Y empiezan a aparecer algunas contribuciones relacionadas con la planificación de espacios litorales, la gestión integrada de zonas costeras, y la gobernanza de la pesca y de los océanos. Sobre puertos y actividad portuaria escriben entre otros Manuel de Terán (1947), Justiniano García Prado (1950), Julia López Gómez (1955, 1973, 1975), Joaquín Bosque Maurel (1952, 1963), Matilde Ruíz (1966), Francisco Quirós Linares (1980), Ramón María Alvargonzález Rodríguez (1983, 1985, 1986), José Ramón Navarro Vera (2004), Jesús Miras Araujo (2004), Alfredo Morales (2004) o Carmen Delgado Viñas (2010). Podemos citar algunos estudios relacionados con la pesca, como los de Nicolás Ortega y Florencio Zoido (1972), J. Benito Arranz (1975, 1987), Diego Compán Vázquez (1975, 1982), Knut Bjorn Londkvist y Rubén Lois (2000), Xosé M. Santos (2001a) o Arnt Fløysand y Aslaug Sæether (2007). Con respecto al estudio de la gobernanza de los mares y de la pesca, o sobre la gestión de puertos y espacios litorales han escrito fundamentalmente Juan Manuel Barragán Muñoz (1990, 1994, 2010) en la Universidad de Cádiz, y Juan Luis Suárez de Vivero, junto con Juan Carlos Rodríguez Mateos y David Florido del Corral, en la Universidad de Sevilla (2008, 2010). Pasamos a continuación a detallar estos aspectos, concretando las contribuciones presentes en cada revista.

\subsection{Estudios Geográficos}

Esta revista publicaba en el año 2015 su número 279. Del análisis de la producción a partir del primer número, podemos concluir que es la que recoge un mayor número de artículos relacionados con las actividades marítimas y pesqueras. Tanto en valores absolutos, porque constituye la primera revista geográfica en castellano, publicada desde 1940, como en términos relativos. Hemos considerado los 1429 artículos publicados en setenta y cinco años para realizar una aproximación bibliométrica. Existen 21 aportaciones en forma de artículo relacionadas con la temática marina o pesquera, considerada en un sentido amplio. Si además tenemos en cuenta las breves presentaciones incluidas en el apartado reservado en esta revista a "Noticias y Comunicaciones" podemos sumar cinco más, lo que parece en conjunto una pobre contribución a estos temas en el conjunto de números publicados. En cuanto a la temática de los 21 artículos podemos establecer dos grandes subapartados. En primer lugar, los más numerosos son los estudios sobre puertos y función portuaria, tanto a través de aproximaciones globales al sistema portuario español como, en la mayoría de las ocasiones, a través de estudios de caso. Se trata de análisis sobre puertos, actividad portuaria, y aspectos específicos de la actividad económica 
desarrollada en los mismos, y suman un total de 14. Esta línea de trabajo es iniciada en la revista por Manuel de Terán, con un trabajo dedicado al puerto de Santander (Terán, 1947), que continúa con la difusión en el siguiente número de una reseña sobre el mismo puerto (Sermet, 1948). También podemos encontrar estudios sobre el puerto de Gijón-Musel (García, 1950), de Bermeo (Doumenge, 1958), de La Garrucha (Ortega \& Zoido, 1972), Vigo (Rodríguez, 1980), y del Grau de Valencia (Roselló, 1990). Resulta de gran interés la serie de estudios publicada por Julia López Gómez, analizando los puertos de Alicante en 1955, de Barcelona en 1973 y de Benicarló en 1975. A su vez, y con una visión más global, Joaquín Bosque Maurel, realizaría dos estudios centrados en las actividades portuarias en España, que serán publicados en 1952 y 1963. En el año 1990, Juan Manuel Barragán Muñoz, Catedrático de la Universidad de Cádiz, presentaría un trabajo sobre la Integración territorial del puerto Bahía de Cádiz, donde ya se avanzaba el tema de trabajo que ha destacado en su producción científica posterior, centrada en la Gestión Integrada de Áreas Litorales. Este autor volvería a publicar en esta misma revista, ya en el año 2010, otro artículo sobre gestión de espacios y recursos costeros en España.

En segundo lugar nos encontramos un total de 12 estudios de temática muy variada, centrados en aspectos relacionados con la comercialización de pescado (López, 1953; Espinosa y Sánchez, 2013), y la caracterización de algunas actividades del sector como la pesca -a escala estatal (Benito, 1975), por ámbitos (Kruiderink, 1967), caladeros (García, 1963), o por especies objetivo (Muro \& Urteaga, 1993; Utanda, 2003)-, la miticultura (Veiga, 1958), y la industria conservera (Quirós, 1966). Los estudios de carácter biológico sobre determinadas pesquerías (López, 1960), y los de enfoque demográfico sobre comunidades pesqueras (Viruela, 1993a) contribuyen a la diversidad de temas tratados. Por último, destacan las investigaciones relacionadas con los conflictos entre Estados por la soberanía y el aprovechamiento de las aguas jurisdiccionales, que tratan en concreto los problemas con Marruecos (Oya, 1975) y Canadá (Santos, 2001a).

\subsection{Boletín de la Asociación de Geógrafos Españoles}

Resulta muy escasa la presencia en esta revista de contribuciones relacionadas con el mar y las actividades a él vinculadas. En los 69 números publicados hasta 2015 se han presentado 885 contribuciones, entre las que solo se encuentran tres artículos sobre con la temática analizada.

Entre las aportaciones presentes aparece en primer lugar, desde el punto de vista cronológico, una centrada en el papel de las infraestructuras portuarias en la ordenación, planificación y gestión del espacio litoral (Barragán, 1994). La segunda, escrita por dos geógrafos noruegos, aborda las relaciones de género y la división del trabajo en una comunidad pesquera de la provincia de $A$ Coruña (Fløysand \& Sæether, 2007). Y por último, en el año 2008, se publica un trabajo que se detiene en los pasos dados por la Unión Europea para afrontar los retos planteados por la Gestión Integral de Zonas Costeras (Tros-de-llarduya, 2008). A estos artículos, podemos sumar uno 
centrado en las actividades extractivas en la Región de Murcia, donde se dedica un breve análisis a la pesca (Morales, 2004b).

El Boletín de la Asociación de Geógrafos Españoles ocupa uno de los primeros lugares entre las revistas españolas de Geografía en índices de impacto nacionales e internacionales. Y resulta muy relevante que en uno de los principales medios de difusión de la investigación geográfica en España encontremos una producción tan exigua, en parte elaborada por geógrafos extranjeros. Podemos apreciar que no se desarrolla ninguna línea de investigación definida, solo aparecen estos trabajos dispersos en el conjunto de la producción global de la revista.

\subsection{Ería}

Hasta el año 2015 se han publicado 98 números, y 587 artículos, entre los que podemos encontrar cinco relacionados con las actividades pesqueras o marítimas. El primero de ellos lo encontramos en el número uno, de 1980, dedicado al estudio del puerto de Gijón (Quirós, 1980). A partir de este momento la mayor parte de las contribuciones se integran en una línea de investigación, desarrollada por Ramón María Alvargonzález Rodríguez, catedrático de Geografía de la Universidad de Oviedo, y centrada en el sistema portuario español, y el análisis de sus características, las funciones y morfología (Alvargonzález, 1983, 1985, 1986). Por último, tenemos que citar un estudio comparativo entre los sistemas pesqueros de Galicia y Noruega (Borj \& Lois, 2000).

\subsection{Anales de Geografía}

Esta revista dedica una especial atención al territorio de la Comunidad de Madrid, lo que puede justificar una menor presencia de los temas marítimos entre los artículos publicados. Hasta 2015 se habían publicado 35 volúmenes, 45 números y 749 contribuciones. Destacamos un único artículo que analiza la significación preindustrial e industrial de la pesca, y su dimensión en la flota andaluza del Atlántico (Benito, 1987).

\subsection{Investigaciones Geográficas}

Entre 1983 y 2015 se publicaron 64 números de esta revista, editada por el Instituto Universitario de Geografía de la Universidad de Alicante. En conjunto, suman 654 aportaciones, excluyendo del análisis las recensiones. De este total, solo cinco se dedican al estudio de la pesca, los puertos, o la ocupación del dominio público marítimo en España. En concreto, dos realizan un análisis de la actividad pesquera desde una perspectiva histórica, a través de estudios de caso en la Comunidad Valenciana, en la Baja Edad Media (Hinojosa, 1995) y en el siglo XIX (Viruela, 1995a). Las otras tres contribuciones son artículos relativos a los puertos valencianos (Navarro, 1994), el puerto de Cartagena (Morales, 2004); y el puerto de A Coruña (Mirás, 2004). 


\subsection{Documents d'Anàlisi Geogràfica}

Revista publicada desde 1982, en el año 2015 apareció el volumen 61, y entre toda su producción no hemos encontrado ningún trabajo relativo a las actividades pesqueras. La revisión de 659 contribuciones nos ha permitido constatar que entre los numerosos artículos publicados desde una perspectiva de género no aparece ningún estudio sobre el trabajo de la mujer en las comunidades costeras, ni sobre el papel fundamental que ha jugado en la modernización de la actividad marisquera, y en el mantenimiento de las actividades pesqueras como motor de desarrollo económico en pequeñas comunidades litorales.

Existen numerosos estudios sobre los problemas de cuantificación del trabajo de las mujeres y su infravaloración en las estadísticas oficiales. Abundan los análisis del trabajo de la mujer en el ámbito rural, pero están centrados fundamentalmente en tareas agropecuarias. Y resulta muy llamativa la ausencia de estudios de género centrados en el mundo del mar, donde la división del trabajo por sexo ha estado, y sigue estando, tan presente en todas las actividades del sector.

\subsection{Scripta Nova}

Esta publicación de la Universidad de Barcelona acepta trabajos procedentes de las Ciencias Sociales y específicamente de la Geografía. Se dirige fundamentalmente a un público de habla catalana, española y portuguesa, aunque también acepta trabajos en otras lenguas. Se publica un número anual desde 1997, y en el año 2015 apareció el 19. Además existen una serie de volúmenes extraordinarios, de carácter temático, publicados entre los años 2011 y 2015. En el conjunto de estos volúmenes, ordinarios y extraordinarios, se recogen 551 contribuciones. Debemos aclarar que entre 1999 y 2008, y posteriormente en 2010, 2012 y 2014 también se han publicado una serie de números a partir de los contenidos presentados en los sucesivos coloquios internacionales de Geocrítica. Al ser contribuciones a congreso, estos volúmenes no se han analizado, y por tanto las aportaciones allí recogidas no se han tenido en cuenta en el cómputo final. Así, entre los más de 500 artículos publicados a los largo de 19 años no aparece ninguno centrado propiamente en análisis de aspectos socioeconómicos vinculados a las actividades pesqueras.

Podemos destacar, por un lado, un artículo que analiza las infraestructuras de transporte y su relación con la morfología de las ciudades portuarias españolas (Delgado, 2010). Por otro, las tres aportaciones relacionadas con la jurisdicción sobre espacios marítimos, a partir de estudios de caso centrados en el Archipiélago canario (Martín, 2005), y en Canadá (Suárez de Vivero et al., 2010). Y con la gobernanza de la pesca, en sus escalas global y local (Suárez de Vivero et al., 2008) cuya autoría corresponde al citado catedrático de la Universidad de Sevilla, junto con otros profesores de los departamentos de Antropología Social y Geografía Humana de la misma universidad. 


\subsection{Cuadernos de Geografía.}

Publicada por la Universidad de Valencia, en 2015 aparece el número 97. Desde su creación en 1964 en esta revista han aparecido 647 aportaciones, de los cuales solo dos se centran en una temática pesquera. En concreto son dos artículos del mismo autor, relativos, en un caso, a la pesca de arrastre en el litoral valenciano (Viruela, 1993b), y en el otro, al análisis de las estrategias de adaptación puestas en marcha por las comunidades de pescadores frente a la crisis de las pesquerías (Viruela, 1995b).

En relación a la geografía portuaria, se encuentran cuatro artículos que estudian los principales puertos valencianos, en concreto la competencia y especialización de los más grandes (Ponce, 1998), el caso del puerto de Valencia (Segrelles \& Gómez, 1997; Sanchís \& Piqueras, 2003), y el de Denia (Ruíz, 1966). En este último caso resulta muy interesante que, en el análisis de Matilde Ruiz Gallego sobre este puerto alicantino, se incluye un estudio detallado sobre la pesca, la principal actividad del puerto en ese momento. Se detallan embarcaciones, artes de pesca, capturas, empleo y comercialización, acompañadas de datos estadísticos y de abundantes gráficos e ilustraciones.

\subsection{Cuadernos Geográficos.}

El primer número de la Revista Cuadernos Geográficos de la Universidad de Granada apareció en 1971, y en el año 2015 se publicó el volumen 54. A lo largo de estos 44 años han aparecido 586 contribuciones centradas en el análisis de la provincia de Granada, del ámbito regional andaluz, pero también abundan los estudios a escalas mayores, de ámbitos peninsulares pero también latinoamericanos e internacionales.

Hemos contabilizado tres contribuciones relacionadas con el estudio de las actividades pesqueras, dos de ellas son artículos firmados, en 1975 y 1982, por Diego Compán Vázquez, del Departamento de Análisis Geográfico Regional y Geografía Física de la Universidad de Granada. En el primer trabajo se realiza un estudio general de la pesca en España, de su importancia económica, del empleo generado y de su incidencia en las ramas industriales. Se analizan los sistemas de capturas y aparejos empleados, los problemas socio laborales, y las dificultades y conflictos existentes en el acceso a caladeros extranjeros (Compán, 1975). En el segundo artículo el ámbito de estudio se restringe a la flota andaluza y se centra en aspectos relacionados con el acceso a caladeros de pesca tradicionales, en especial a los de soberanía marroquí (Compán, 1982). La última aportación sobre esta temática aparecida en esta revista data de 1984 y describe los sistemas y artes de pesca empleados en la pesca artesanal desarrollada en el Cabo de Gata (Márquez, 1984). 


\subsection{Semata}

Por último se ha considerado la producción científica de la revista Semata, publicación anual de la Facultad de Geografía e Historia de la Universidad de Santiago de Compostela. La elección de esta revista, en lugar de Xeográfica, la propia del Departamento de Geografía de esta universidad, viene condicionada porque aquella publicó su último número en 2007.

Los 27 números aparecidos entre 1988 e 2015 tienen carácter monográfico, y gran parte de los estudios una escala marcadamente regional. El número 25, de 2013, lleva por título "El mar: una mirada transversal desde las ciencias sociales y las humanidades". Coordinado por Xosé M. Santos Solla y por Alberto Martí Ezpeleta, profesores de Departamento de Geografía de esta universidad, vino a cubrir el vacío que existía en relación a esta temática.

Además del citado número monográfico, en el resto de las contribuciones publicadas por la revista solo aparece una más relacionada con la pesca, en 1997, en el número dedicado a "Espacios rurales y sociedades campesinas". Se trata de un artículo donde se realiza un estudio crítico de las estadísticas pesqueras (Pérez, 1997).

Tabla 1. Contribuciones en revistas geográficas españolas hasta el fin de 2015

\begin{tabular}{|l|c|c|c|c|c|}
\hline & $\begin{array}{c}\text { Números } \\
\text { publicados } \\
(\mathbf{2 0 1 5 )}\end{array}$ & Años & $\begin{array}{c}\text { Total } \\
\text { contribuciones } \\
(\mathrm{T})\end{array}$ & $\begin{array}{c}\text { Asuntos } \\
\text { marítimos } \\
(\mathrm{M})\end{array}$ & $\begin{array}{c}\text { M/T } \\
\text { (\%) }\end{array}$ \\
\hline Estudios Geográficos & 279 & $1940-2015$ & 1429 & 26 & 1,8 \\
\hline Boletín AGE & 69 & $1984-2015$ & 885 & 3 & 0,3 \\
\hline Ería & 98 & $1980-2015$ & 587 & 5 & 0,9 \\
\hline Anales de Geografía & 45 & $1981-2015$ & 749 & 1 & 0,1 \\
\hline Investigaciones Geográficas & 64 & $1983-2015$ & 654 & 5 & 0,9 \\
\hline Documents d'Anàlisi Geogràfica & 73 & $1982-2015$ & 659 & 0 & 0 \\
\hline Scripta Nova & 19 & $1997-2015$ & 551 & 4 & 0,7 \\
\hline Cuadernos de Geografía & 97 & $1964-2015$ & 647 & 6 & 0,9 \\
\hline Cuadernos Geográficos & 54 & $1971-2015$ & 586 & 3 & 0,5 \\
\hline Semata & 27 & $1987-2015$ & 463 & 15 & 3,2 \\
\hline
\end{tabular}

Fuente: elaboración propia a partir de las revistas citadas

Tal y como se ha comentado, se consideró oportuno ampliar el ámbito de estudio a la Revista Española de Estudios Agrosociales y Pesqueros. Editada por el Ministerio de Agricultura, Alimentación y Medio Ambiente desde 1998 tiene una periodicidad fundamentalmente cuatrimestral. De los casi 300 trabajos publicados, solo 9 tienen una temática relacionada con la pesca, y todos ellos tienen como autores o coautores a tres catedráticos de economía aplicada de la Universidad de Vigo. Se trata de los especialistas en economía pesquera Manuel Varela Lafuente y Juan Carlos Surís Regueiro, que son coautores en 5 de estos trabajos, y María Dolores Garza Gil, coautora en 4 de ellos. Todos los artículos dentro de esta temática son escritos por economistas, 
excepto en un único caso, constituido por el artículo publicado por David Florido del Corral, antropólogo social de la Universidad de Sevilla (Florido, 2005). No existe ningún artículo centrado en el análisis de la actividad pesquera firmado por un geógrafo.

Este análisis se completó con el estudio de tres monográficos dedicados al estudio de la pesca: el $n^{\circ}$ 546, de 1979, de la revista Información Comercial Española; el n 4, de 1984, de la revista Situación; y el n 99, de 1985, de la revista El Campo. Entre los trabajos publicados en estos 3 monográficos solo existe una contribución aportada por un geógrafo (Oya, 1985), relativa al nuevo marco de las relaciones pesqueras internacionales a partir de la entrada en la Unión Europea. La gran mayoría de los trabajos son presentados por economistas.

Por último, nos parece adecuado citar dos revistas, ya desaparecidas, por la relevancia que en su día tuvieron para la difusión de la producción geográfica universitaria gallega. Nos referimos a la ya citada Xeográfica, revista de xeografía, territorio e medio ambiente, de la se publicó un número anual entre 2001 y 2007, y en la que apareció un estudio sobre los puertos de interés general de Estado en Galicia (Díaz, 2003), que supone la única referencia a las actividades marítimas en esta revista. Y, en segundo lugar, la revista Terra, de la Sociedade Galega de Xeografía, de la que se llevan publicados seis números, el último en 2002, y donde nunca se ha publicado ningún artículo relacionado con la pesca o el mundo del mar en Galicia. Esta ausencia de trabajos en las dos revistas de referencia para los geógrafos en la comunidad gallega, son reveladores del escaso interés que ha tenido esta línea de investigación, que por el contrario ha conseguido gran desarrollo desde la perspectiva economía aplicada.

\section{Conclusiones}

Al analizar el conjunto de los trabajos presentados en las citadas revistas, y en especial aquellos relacionados con el estudio del mar y de las actividades marítimas, se obtienen varias conclusiones. La más inmediata es la existencia de un número escaso de contribuciones centradas en las actividades pesqueras, y la mayor importancia relativa que adquieren los artículos dedicados al análisis de los puertos y de la función portuaria de las ciudades litorales en España, gracias a la trayectoria investigadora de algunos geógrafos especializados en esta temática. En los últimos decenios empiezan a aparecer en algunas revistas artículos sobre política y gobernanza aplicada a pesca y océanos, y sobre gestión integrada de espacios litorales, pero estos primeros trabajos aún no son los suficientemente numerosos como para crear una tendencia dentro de la investigación geográfica.

En las revistas científicas de geografía españolas analizadas no se publican trabajos sobre el impacto territorial de las políticas pesqueras a diferentes escalas, ni autonómica, ni estatal, ni europea. Por el contrario, encontramos abundante bibliografía sobre la Política Agraria Común, 
sobre sus efectos en el medio rural español, y sobre la aplicación de la gestión de base local de los fondos comunitarios. Pero no vemos un interés similar por la política sectorial de pesca de la Unión Europea, por sus consecuencias en el territorio y en los subsectores derivados, ni sobre la gestión y distribución de los fondos públicos y privados en las comunidades costeras españolas. Ni para la geografía económica, ni para la geografía rural, el mar y las actividades a él vinculadas han supuesto un ámbito de interés. No existen aportaciones en el ámbito de la geografía política sobre las aspiraciones recientes de los estados para ampliar su jurisdicción en el mar y en los fondos marinos. La geografía de la población no se ha ocupado de analizar los movimientos migratorios relacionados con la actividad pesquera. Se echan de menos trabajos sobre los trasvases humanos como consecuencia de los acuerdos pesqueros, proliferación de empresas mixtas pesqueras, y la expansión de la flota industrial española por aguas de todo el mundo. Por otra parte, no se aborda el análisis geográfico de la división del trabajo en comunidades costeras desde una perspectiva de género, aun cuando algunas de las revistas analizadas se han especializado en este enfoque. Queda cuando menos por analizar el empoderamiento de las mujeres y su relación con la profesionalización del marisqueo, y con el mantenimiento de la pesca artesanal y sostenible, o su papel en la diversificación de las economías costeras. No encontramos en las revistas analizadas aportaciones desde la geografía del turismo al análisis de las nuevas actividades relacionadas con el turismo pesquero, que han experimentado un gran auge en décadas recientes gracias a la llegada de fondos europeos. Gran parte de estos dineros públicos han servido para poner en valor el patrimonio cultural material e inmaterial vinculado a la cultura marinera lo que ha favorecido la aparición de numerosas empresas relacionadas con este tipo de turismo que han modificado la oferta en pequeñas comunidades costeras, altamente dependientes de la pesca, y que están pendientes de estudiar.

El estudio de los mares está ausente de la mayor parte de la literatura geográfica contemporánea, y esta característica, unida a su justificación por los problemas generados con las fuentes de estudio, suponen una línea de investigación en sí misma. Las causas de esta situación son complejas y variadas, y no es menor la importancia de la consideración del mar como simple superficie de tránsito en contraposición a la tierra hasta bien avanzado el siglo XIX. Y aunque a lo largo del siglo XX fue apareciendo el interés por los transportes marítimos, los complejos portuarios y su ordenación, la investigación sobre la polución de los mares y sobreexplotación de los recursos tiene poca visibilidad en las revistas científicas españolas. Pero existen otras motivaciones sumadas a la anterior y no de menor peso. La escasa valoración que tradicionalmente se le ha dado la actividad pesquera, la dificultad para su estudio por la ausencia o inadecuación de las estadísticas sectoriales, o el hecho de que tenga especial relevancia sobre todo en las comunidades más periféricas de España, parecen justificar el escaso interés de la geografía. A ello hay que añadir la carencia de tradición entre los geógrafos, la falta de maestros y de escuelas de referencia en los 
estudios sobre pesca, que suponen un motivo más entre los que contribuyen a mantener esta situación.

El insuficiente nivel de análisis de las actividades relacionadas con el mar y con la actividad marítima es general en todas las regiones españolas, incluso en aquellas en donde tienen un gran peso socioeconómico. Se han hecho acercamientos aislados a esta cuestión, pero no se ha desarrollado una línea de investigación centrada en el sector. Este campo de estudio se ha dejado fundamentalmente en mano de economistas, sociólogos o antropólogos, y solo pocos geógrafos en España tienen ésta como su principal línea de trabajo.

Declaración responsable: Las/os autoras/es declaran que no existe ningún conflicto de interés en relación a la publicación de este artículo. A partir de una selección inicial de las revistas a estudiar, realizada por ambas/os autoras/es, la primera autora se ocupó de realizar el estudio del contenido de las mismas. Posteriormente, ambas/os autoras/es han realizado conjuntamente el análisis de los resultados y la redacción de las conclusiones. 


\section{Bibliografía}

Alvargonzález Rodríguez, R. M. (1983). Los rasgos generales del sistema portuario español a través del tráfico de mercancías. Ería, 4, 101-106.

Alvargonzález Rodríguez, R. M. (1985). Funciones y morfología de los puertos españoles. Ería, 8, $5-60$.

Alvargonzález Rodríguez, R. M. (1986). Evolución y actividad de la Marina Mercante Nacional en la década de 1970. Ería, 10, 139-143.

Benito Arranz, J. (1975). La pesca marítima en España y su estudio geográfico. Estudios Geográficos, 36(138-139), 31-62.

Benito Arranz, J. (1987). Significación preindustrial e industrial de la pesca y sus concomitancias en las faenas de la flota andaluza del atlántico. Anales de Geografía de la Universidad Complutense, 7, 229-239.

Barragán Muñoz, J. M. (1990). Integración territorial del puerto Bahía de Cádiz. Estudios Geográficos, 51(198), 7-32.

Barragán Muñoz, J. M. (1994). Las infraestructuras portuarias en la ordenación, planificación y gestión del espacio litoral. Boletín de la Asociación de Geógrafos Españoles, 19, 5-16.

Barragán Muñoz, J. M. (2010). La gestión de los espacios y recursos costeros en España. Política e instituciones de una legislatura (2004-2008). Estudios Geográficos, 71(268), 39-65.

Benito Arranz, J. (1975). La pesca marítima en España y su estudio geográfico. Estudios Geográficos, 36(138-139), 31-62.

Borj Lindkvist, K. Y Lois González, R. C. (2000). Aspectos regionales de las pesquerías de Galicia y Noruega. Ería, 51, 69-78.

Bosque Maurel, J. (1952). Funciones económicas de los puertos españoles de la península. Estudios Geográficos, 13(48), 569-577.

Bosque Maurel, J. (1963). Las actividades portuarias en España. Estudios Geográficos, 24(93), $602-610$.

Compán Vázquez, D. (1975-1976). La pesca marítima en España. Cuadernos Geográficos, 5-6, $87-176$.

Compán Vázquez, D. (1982). Dinámica locacional reciente de los caladeros de pesca de la flota radicada en Andalucía. Cuadernos Geográficos, 12, 87-102. 
Delgado Viñas, C. (2010). Entre el puerto y la estación. La influencia de las infraestructuras de transporte en la morfología de las ciudades portuarias españolas (1848-1936). Scripta Nova, 14, $323-339$.

Díaz Fernández, J. A. (2003). Los puertos de interés general del Estado de Galicia ante el reto del s. XXI. Luces y sombras de una política portuaria dinamizadora de los espacios litorales. Xeográfica, 3, 53-78.

Doumenge, F. (1958). La pesca en Bermeo. Estudios Geográficos, 19(72), 365-380.

Durán Villa, F. R., Ferrás Sexto, C., Lois González, R. C., \& Santos Solla, X. M. (1998). A propósito del Atlántico como tema de reflexión geográfica. Aproximación temática y bibliográfica a una cuestión escasamente tratada por la Geografía española. Boletín de la Asociación de Geógrafos Españoles, 25, 105-122.

Espinosa Seguí, A., \& Sánchez Hernández, J. L. (2013). El mercado español del bacalao en salazón: una aproximación regional desde la geografía de las convenciones. Estudios Geográficos, 74(275), 439-467.

Florido del Corral, D. (2005). Más allá de las cifras del sector pesquero: marco de economía política y estrategias de los pescadores artesanales andaluces del golfo de Cádiz. Revista española de estudios agrosociales y pesqueros, 208, 171-196.

Fløysand, A., \& Sæether, A. (2007). Globalización, comunidades pesqueras y construcción de género: el caso de Muros (Galicia). Boletín de la Asociación de Geógrafos Españoles, 45, 3148.

García Cabrera, C. (1963). El banco pesquero canario-sahariano. Estudios Geográficos, 24(91), 147-197.

García Prado, J. (1950). El tráfico del puerto Gijón-Musel en el año 1949. Estudios Geográficos, 17(41), 711-714.

Hinojosa Montalvo, J. R. (1995). Comercio, Pesca y Sal en el Cap de Cerver (Orihuela) en la Baja Edad Media. Investigaciones geográficas, 14, 191-204.

Kruiderink, A. (1967). Algunos aspectos de la pesca mediterránea en España. Estudios Geográficos, 28(106), 89-113.

Lois González, R. C. (1999). La pesca: actividad económica y organización espacial. In R. Galdós Urrutia, \& E. Ruiz Urrestarazu (Coords.), Postproductivismo y Medio Ambiente. Perspectivas geográficas sobre el espacio rural (pp. 113-175). Presented at the IX Coloquio de Geografía Rural. Vitoria-Gasteiz: Departamento de Agricultura y Pesca. 
López Gómez, A. (1953). El abastecimiento de pescado en Madrid. Estudios Geográficos, 14(53), 527-593.

López Gómez, J. (1955). El puerto de Alicante. Estudios Geográficos, 16(60), 511-583.

López Gómez, J. (1960). La crisis sardinera en Vigo: estudios biológicos. Estudios Geográficos, 21(80), 414-416.

López Gómez, J. (1973). El puerto de Barcelona. Estudios Geográficos, 34(131), 429-438.

López Gómez, J. (1975). El puerto de Benicarló. Estudios Geográficos, 36(140-141), 609-648.

Márquez Úbeda, J. (1984-1985). Aportación al estudio de la pesca con artes menores en la costa almeriense. Cabo de Gata. Cuadernos Geográficos, 14, 101-122.

Martín Lou, M. A., Bodega. I., Cebrián de Miguel, J. A., \& Jiménez Royo. A. (2013). Las publicaciones de geógrafos españoles en revistas de Geografía españolas. In T. Lasanta, \& J. Martín Vide (Coords.), La investigación geográfica en España (1990-2012) (pp. 235-248). Madrid: Asociación de Geógrafos Españoles, Instituto Pirenaico de Ecología (CSIC), Centro de Ciencias Humanas y Sociales (CSIC).

Martín Ruiz, J. F. (2005). Los espacios marítimos y el problema de su delimitación en la posición geopolítica del Archipiélago canario. Scripta Nova, 9, 181-204.

Martínez Hernández, C., \& Moreno Muñoz, D. (2013). La pesca como recurso turístico en Mazarrón (Murcia). Estudios turísticos, 196, 113-134.

Mirás Araujo, J. (2004). El tráfico mercantil portuario en una etapa de transición: A Coruña, 19131960. Investigaciones geográficas, 33, 119-140.

Morales Gil, A. (2004). Trascendencia territorial del puerto de Cartagena. Investigaciones geográficas, 33, 107-118.

Morales Gil, A. (2004b). Significado y valor de las actividades extractivas en la Región de Murcia. Boletín de la Asociación de Geógrafos Españoles, 38, 71-84.

Muro Morales, J. I., \& Urteaga González, L. (1993). Una serie histórica sobre producción pesquera: las almadrabas de la bahía de Cádiz (1525-1763). Estudios Geográficos, 54(211), 323-354.

Navarro Vera, J. R. (1994). Aproximación analítica a los sistemas portuarios y su aplicación a los puertos valencianos. Investigaciones geográficas, 12, 147-162.

Ortega Cantero, N., \& Zoido Naranjo, F. (1972). Garrucha: la pesca de bajura en un pequeño puerto mediterráneo. Estudios Geográficos, 33(127), 313-354. 
Oya, J. J. (1975). Los apresamientos de buques de pesca españoles por la Armada marroquí (febrero 1973-mayo 1974): Estudio geográfico de un contencioso pesquero. Estudios Geográficos, 36(140-141), 901-950.

Oya, J. J. (1985). El nuevo marco de las relaciones pesqueras internacionales. El campo, 99, 2733.

Pardellas, X., \& Padín, C. (2013). La nueva demanda combinada de turismo litoral y turismo pesquero: motivaciones y efectos. Cuadernos de turismo, 32, 243-258.

Pérez Iglesias, M. L. (1996). Geographical Research on Fishing Activities in Spain. In A. López Ontiveros, \& F. Molinero Hernando, From Traditional Countryside to Postproductivism: Recent Trends in Rural Geography Research in Britain and Spain (pp. 205-2012). Primer Simposium de Geógrafos rurales británicos y españoles, organized by the Asociación de Geógrafos Españoles.Leicester, September 8-13.

Pérez Piernas, P., \& Espejo Marín, C. (2012). La pesca como factor de desarrollo del turismo sostenible. El caso de Águilas (Murcia). Cuadernos de turismo, 30, 267-284.

Pérez Sánchez, J. A. (1997). De la pesca y su explotación en la investigación geográfica gallega: un análisis crítico de las estadísticas pesqueras. Semata, 9, 129-142.

Ponce Herrero, G. J. (1998). Competencia y especialización en los grandes puertos valencianos, Cuadernos de Geografía, 63, 195-216.

Quirós Linares, F. (1966). La industria española de conservas de pescado. Estudios Geográficos, 27(104), 505-513.

Quirós Linares, F. (1980). El puerto de Gijón. Ería, 1, 179-221.

Rodríguez Arzúa, J. (1980). El puerto de Vigo, 1900-1975. Estudios Geográficos, 41(160), 237266.

Rosselló i Verger, V. M. (1990). El Grau de Valencia, un puerto difícil. Estudios Geográficos, 51(199-200), 619-634.

Ruíz Gallego, M. (1966-1967). El puerto de Denia. Cuadernos de Geografía, 3-4, 235-267.

Sanchís Deusa, M. C., \& Piqueras Haba, J. (2003). El tráfico marítimo de contenedores. Valencia en la red portuaria mundial. Cuadernos de Geografía, 73-74, 147-182.

Santos Solla, X. M. (2001a). La crisis del fletán negro: Una perspectiva desde la prensa canadiense. Estudios Geográficos, 62(242), 105-138.

Santos Solla, X. M. (2001b). La explotación de los recursos marinos: pesca, acuicultura y marisqueo. In Actas del XV Congreso de Geógrafos Españoles "Los Espacios litorales y 
emergentes" (pp. 213-254). Santiago de Compostela: Servicio de Publicaciones de la Universidad de Santiago.

Segrelles Serrano, J. A., \& Gómez López, J. D. (1997). El Puerto de Valencia y la articulación territorial de su espacio económico. El caso del calzado. Cuadernos de Geografía, 61, 159-168.

Sermet, J. (1948). El puerto de Santander. Estudios Geográficos, 9(33), 637-647.

Suárez De Vivero, J. L. (1979, March). El espacio marítimo en la geografía humana. Geocrítica, 20. Retrieved from http://www.ub.edu/geocrit/geo20.htm

Suárez De Vivero, J. L., Rodríguez Mateos, J. C., \& Florido Del Corral, D. (2008). La gobernanza en la pesca: de lo ecológico a lo ético, de lo local a lo global. Scripta Nova, 12, 256-280.

Suárez De Vivero, J. L., Rodríguez Mateos, J. C., \& Sacchetti, E. (2010). Canadá y el gobierno de los océanos: el espacio marítimo en un Estado descentralizado. Scripta Nova, 14, 310-322.

Terán, M. de (1947). Santander, puerto de embarque para las harinas de Castilla. Estudios Geográficos, 8(29), 746-758.

Tros-De-llarduya Fernández, M. (2008). El reto de la Gestión Integrada de las Zonas Costeras (GIZC) en la Unión Europea. Boletín de la Asociación de Geógrafos Españoles, 47, 143-156.

Utanda Moreno, L. (2003). La pesca de salmón en España (1949-1999). Estudios Geográficos, 64(250), 87-106.

Vázquez, A., Lois, R. C. (2001). The Problem of Fishery Statistics in Spain. International Conference People and the Sea: Maritime research in the social sciences-an agenda for the 21st century. Abstract retrieved from http://www. marecentre.n//people and the sea/documents/abstracts.pdf Veiga González, C. (1958). El cultivo del mejillón en la ría de Vigo. Estudios Geográficos, 19(73), 513-538.

Viruela Martínez, R. (1993a). Dinámica y estructura de la población pesquera valenciana. Estudios Geográficos, 54(213), 695-724.

Viruela Martínez, R. (1993b). Difusió de la pesca del bou en el litoral de Valencia (segles XVIII i XIX). Cuadernos de Geografía, 53, 145-161.

Viruela Martínez, R. (1995a). Expansión y crisis de la actividad pesquera valenciana en el siglo XIX. Investigaciones geográficas, 13, 117-134.

Viruela Martínez, R. (1995b). Las comunidades pesqueras ante la crisis de las pesquerías y el cambio económico. Cuadernos de Geografía, 57, 153-182. 